\title{
Antimicrobial susceptibility patterns of Escherichia coli phylogenetic groups isolated from bovine clinical mastitis
}

\author{
T. Tomazi, ${ }^{*}$ F. M. Coura, † J. L. Gonçalves, ${ }^{*}$ M. B. Heinemann, $\ddagger$ and M. V. Santos ${ }^{* 1}$ \\ *Department of Animal Nutrition and Production, School of Veterinary Medicine and Animal Science, University of São Paulo, \\ Pirassununga 13635-900, SP, Brazil \\ †Departament of Agricultural Science, Federal Institute of Minas Gerais, Campus Bambuí, Fazenda Varginha, Rodovia Bambuí/Medeiros, Km 05, \\ Caixa Postal 05, Bambuí, MG, 38.900-000, Brazil \\ $\ddagger$ Department of Preventive Veterinary Medicine and Animal Health, School of Veterinary Medicine and Animal Science, University of São Paulo, \\ São Paulo 05508-900, SP, Brazil
}

\begin{abstract}
Determination of antimicrobial susceptibility (AMS) of Escherichia coli causing clinical mastitis (CM) according to the phylogenetic groups and its association with descriptors at the cow and herd level may help improve specific strategies for treatment and control of this pathogen in dairy herds. The aims of the present study were to (a) determine the frequency of phylogenetic groups of E. coli isolated from CM in dairy cows, and its association with cow-level descriptors (parity, lactation stage, CM severity, and affected quarter position), housing system, and season; and (b) determine and compare AMS among E. coli phylogenetic groups. A quadruplex PCR method was used to classify E. coli isolates into 1 of the 7 phylogenetic groups. Minimal inhibitory concentrations were determined for 10 antimicrobials, and survival analysis was performed to evaluate the AMS differences among E. coli phylogroups. Most E. coli isolates belonged to phylogroups A (52\%) and B1 (38\%). None of the cow- and herd-level descriptors were associated with the $E$. coli phylogenetic groups. Overall, E. coli isolates were mostly susceptible to ceftiofur (96.8\%), sulfadimethoxine (75.5\%), and cephalothin (74.5\%). Based on the survival analysis, differences in AMS between phylogenetic groups of $E$. coli was observed only for cephalothin, in which strains of phylogroup A were inhibited at lower minimum inhibitory concentration than strains of phylogroup B1. Results of this study indicated low susceptibility of $E$. coli isolates identified from CM to most antimicrobials. In addition, differences in AMS can occur among E. coli phylogenetic groups, although they may be uncommon as they were limited to only one antimicrobial (i.e., cephalothin).
\end{abstract}

Received January 23, 2018.

Accepted April 16, 2018.

${ }^{1}$ Corresponding author: mveiga@usp.br
Key words: antimicrobial susceptibility, bovine intramammary infection, Escherichia coli, phylogeny

\section{INTRODUCTION}

Mastitis is one of the most important diseases affecting dairy cattle, and Escherichia coli is one of the main causes of clinical mastitis (CM) in dairy herds (Oliveira et al., 2013). Escherichia coli strains are mostly commensals; however, pathogenic types were also reported, which were clustered into different pathovars based on clinical data and specific virulence properties (Kempf et al., 2016). Recently, the term mammary pathogenic $E$. coli (MPEC) was proposed to classify pathovars associated with mastitis (Shpigel et al., 2008), although no specific virulence traits have been identified to classify these strains as specifically mastitis-related (Leimbach at al., 2017). Therefore, E. coli causing mastitis appears to be more associated with host and exposure factors, such as cows' health (and immune) status and environmental hygiene (Klaas and Zadoks, 2017), rather than a set of mammary pathogenic strains that affect risk of IMI. As has been shown for many other mastitis-causing pathogens, E. coli has a great intraspecific diversity and can be classified into different phylogenetic groups (Kempf et al., 2016).

Most studies describing the distribution of $E$. coli causing mastitis in phylogenetic groups were performed using the simple triplex PCR method that enables strains of $E$. coli to be assigned into one of the phylogroups A, B1, B2, or D (Clermont et al., 2000). Few reports described the use of the improved quadruplex PCR method in dairy cattle (Kempf et al., 2016), which allows the identification of 7 phylogenetic groups (A, B1, B2, C, D, E, and F; Clermont et al., 2013). Therefore, data on the frequency of groups C, E, and $\mathrm{F}$ causing $\mathrm{CM}$ are scarce in the indexed literature (Kempf et al., 2016). In addition, to our knowledge, studies describing the phylogeny of $E$. coli have not 
been evaluated in isolates of bovine CM from Brazil. The identification of $E$. coli phylogeny may improve the epidemiological understanding of this pathogen in dairy herds, especially in relation to the route of transmission, pathogenicity, virulence factors, and antimicrobial susceptibility (AMS).

The majority of E. coli strains causing bovine mastitis were reported to belong to phylogroups A and B1 (Liu et al., 2014; Keane, 2016). In addition, virulence genes were more often detected in certain phylogroups than others (Suojala et al., 2011; Liu et al., 2014; Zhang et al., 2018). Therefore, considering the potential diversity of genetic properties (e.g., virulence genes) among phylogroups, it can be hypothesized that different groups could be more or less frequent in dairy herds according to characteristics at the cow-level, such as parity, stage of lactation, or even with characteristics that may affect CM occurrence in the herd, such as housing system and season of the year. Furthermore, different $E$. coli strains can cause CM with signs varying from mild to severe, and considering that some virulence factors can be more present in certain phylogenetic group than others, it can be hypothesized that the phylogenetic group may be associated with CM severity.

Clinical mastitis caused by $E$. coli is mostly of short duration, and the cow's immune system is generally competent to spontaneously eliminate mild and moderate infections (Burvenich et al., 2003). Therefore, antimicrobial treatment might not be necessary for most E. coli $\mathrm{CM}$ cases. However, even though interest in the use of management strategies for selective therapy such as on-farm culture is growing in dairy herds (Lago et al., 2011), most farms still do not identify the pathogen causing CM before treatment. Therefore, blanket antimicrobial therapy remains the main strategy for $\mathrm{CM}$ treatment in dairy herds.

The abusive and nonjudicious use of antimicrobials in dairy herds was associated with increased risk of antimicrobial resistance, which has been reported as a significant cause of mastitis treatment failure (Suojala et al., 2013). However, the association between the phylogeny of E. coli causing CM and AMS needs further explanation. Whereas some studies reported an association of AMS and specific phylogenetic groups (Liu et al., 2014), others found no significant association (Keane, 2016). Since blanket treatments of CM are still largely used in dairy herds, a better understanding of the susceptibility of $E$. coli can be used to monitor the efficiency of antimicrobials used for the treatment of IMI caused by this pathogen. Furthermore, the evaluation of $E$. coli susceptibility at the phylogenetic group-level can also serve as a basis for further studies evaluating potential resistance traits of this pathogen causing mastitis.
The objectives of this study were to (1) determine the frequency of phylogenetic groups of $E$. coli strains isolated from CM in dairy cows and its association with the following variables: cow-level descriptors (DIM, parity, position of affected quarter, and CM severity score), housing system and season; and (2) determine and compare AMS among the most prevalent E. coli phylogenetic groups identified from CM.

\section{MATERIALS AND METHODS}

\section{Herd Selection and Bacterial Isolation, Identification, and Storage}

Isolates were selected from a collection of E. coli previously identified by microbiological culture during the data collection of a study on epidemiological characterization of CM in 20 dairy herds of southeastern Brazil (Tomazi, 2017). Herds were conveniently selected based on a client list of the Qualileite Lab (Mastitis and Milk Quality Research Laboratory at University of Sao Paulo, Brazil). Clinical mastitis was identified at the quarter-level upon routine examination of fore-stripped milk immediately before milking by trained farm personnel, and aseptic milk samples were collected from the affected quarter(s) according to guidelines of NMC (1999). After collection, milk samples were frozen and sent in batches to the microbiology laboratory on ice packs for culture or on-farm stored (at approximately $-20^{\circ} \mathrm{C}$ ) until retrieval by researchers. Farm visits by researchers were performed every 14 to $30 \mathrm{~d}$.

During the study period (March 2014 to January 2016), 276 E. coli isolates were identified by microbiological culture of 4,212 individual quarter milk samples collected from 2,637 cows with CM (Tomazi, 2017). Briefly, a $0.01-\mathrm{mL}$ sterile loop was used to plate milk samples onto trypticase soy agar plates (BBL-Becton Dickinson and Co., LePoint de Claix, France) enriched with $5 \%$ bovine blood, and plates were incubated aerobically at $37^{\circ} \mathrm{C}$. Phenotypic features were examined at 24 and $48 \mathrm{~h}$ after incubation and specific biochemical testing was performed to determine bacterial genus, species, or both. All isolates were submitted for $\mathrm{KOH}$ (potassium hydroxide) test. Isolates with positive test reaction were suggestive of gram-negative microorganisms and then were presumptively identified by colony morphology on MacConkey's agar. Identification of $E$. coli isolates was performed using a set of biochemical reactions: sucrose and glucose fermentation, hydrolysis of urea, gas production, motility capacity, indole production, $\mathrm{H}_{2} \mathrm{~S}$ production, L-tryptophan deaminase, and lysine reaction.

A milk sample was defined as culture negative if no colonies were observed on the streaking field of the agar 
plate after $48 \mathrm{~h}$ of incubation. On the other hand, a milk sample was defined as culture positive if at least one colony of $E$. coli was observed in the streaking field of the agar plate (Dohoo et al., 2011). A milk sample was defined as contaminated if $>2$ different colony types were present in the streaked field of the agar plate.

In addition, all isolates identified as E. coli by microbiological culturing were confirmed using MALDI-TOF MS according to the method described by Barreiro et al. (2010). Only E. coli isolates identified with MALDI scores identification at species level $(>2.0)$ were selected for this study. After microbiological culturing, a total of $177 \mathrm{E}$. coli isolates were stored at $-80^{\circ} \mathrm{C}$ in vials containing $2 \mathrm{~mL}$ of brain heart infusion (Becton, Dickinson and Company/BBL, Le Pont Claix, France) broth supplemented with $10 \%$ glycerin. For the present study, $100 \mathrm{E}$. coli isolates were selected after meeting the following inclusion criteria: (1) isolated from a new case of CM (occurring in the same quarter of the same cow at least $14 \mathrm{~d}$ apart); (2) identified from cultures with identification of a single pathogen instead of mixed cultures; and (3) having a pure growth (without contamination) in the re-cultivation performed before phylotyping.

Selection of isolates was performed with the intention to include in the study strains identified in all herds. Thus, all strains were selected for herds with less than 12 isolates; whereas, for herds with $\geq 12$ isolates, strains were randomly selected using the RAND function of Excel software (2010, Microsoft Office Corporation, Redmond, WA).

\section{DNA Extraction}

All E. coli isolates were cultured overnight on blood agar plates at $37^{\circ} \mathrm{C}$ under aerobic conditions. The DNA extraction of $E$. coli isolates was performed using an adaptation of the method described by Fan et al. (1995). In our study, PBS (pH 7.2) was substituted with TrisEDTA buffer $(10 \mathrm{~m} M$ Tris $\mathrm{HCl}, 5 \mathrm{~m} M$ EDTA, $\mathrm{pH}=$ 8.0). After DNA extraction, the lysates were kept frozen $\left(-20^{\circ} \mathrm{C}\right)$ until phylogenetic grouping.

\section{Phylogenetic Group Determination}

Escherichia coli isolates were assigned to 1 of the 7 phylogroups (A, B1, B2, C, D, E, or F) based on amplification of $c h u A, y j a A$, $\operatorname{arp} A$, and $\operatorname{trp} A$ genes and the TSPE4.C2 DNA fragment by PCR, according to Clermont et al. (2013). The reference E. coli strain ATCC 25922 was used as quality control throughout the procedure.

All PCR reactions were carried out in a $20 \mu \mathrm{L}$ final volume that contained the following: $100 \mathrm{ng}$ of DNA,
$0.2 \mathrm{~m} M$ of each deoxynucleotide triphosphate, $1 \times$ Green GoTaq Reaction Buffer (Promega), 2 U of GoTaq DNA polymerase (Promega), and $1 \mu \mathrm{L}$ of each primer, except for the primers of $\operatorname{arp} A$ gene in the quadruplex analysis, of which $2 \mu \mathrm{L}$ was used. The PCR reactions were performed under the following conditions: denaturation for $4 \mathrm{~min}$ at $94^{\circ} \mathrm{C}, 30$ cycles of $20 \mathrm{~s}$ at $94^{\circ} \mathrm{C}, 20 \mathrm{~s}$ at $57^{\circ} \mathrm{C}$ (group E) or $59^{\circ} \mathrm{C}$ (quadruplex and group C), and 25 s at $72^{\circ} \mathrm{C}$, and a final extension step of 5 min at $72^{\circ} \mathrm{C}$ (Clermont et al., 2013).

Ten microliters of the amplified product was electrophoresed in $1.5 \%$ agarose gel with $1 \times$ Tris-borateEDTA buffer stained with $0.1 \mu \mathrm{g} / \mu \mathrm{L}$ of ethidium bromide. Images of gel were taken under UV light using a photo-documentation system. A 100-bp DNA ladder (Thermo Fisher Scientific Inc., Pittsburgh, PA) was used to evaluate whether specific bands were present and to compare size of the bands generated in the electrophoresis.

\section{Antimicrobial Susceptibility Testing}

The MIC of selected antimicrobials against E. coli isolates was tested using the mastitis panel of a commercial broth microdilution test (CMV1AMAF, Sensititer, TREK Diagnostic Systems Inc., Cleveland, OH), following the manufacturer's guidelines. For each batch of microorganism tested, the CLSI (2013)-recommended quality control strains of E. coli ATCC 25922 and Staphylococcus aureus ATCC 29213 were also evaluated. The antimicrobial agents and dilution ranges tested for each are presented in Table 1.

The concentrations of antimicrobial that inhibited $50 \%\left(\mathbf{M I C}_{50}\right)$ and $90 \%\left(\mathbf{M I C}_{\mathbf{9 0}}\right)$ of $E$. coli isolates were calculated for each antimicrobial. Where interpretive cut-points for MIC have been established by CLSI (2013) for E. coli and antimicrobials evaluated, these were used to classify each isolate as susceptible, intermediate, or resistant. Isolates classified as intermediate were interpreted as resistant.

Table 1. Antimicrobial agents and their dilution ranges used in the susceptibility test of Escherichia coli isolates identified from clinical mastitis $(\mathrm{CM})$

\begin{tabular}{lc}
\hline Antimicrobial agent & MIC dilution range $(\mu \mathrm{g} / \mathrm{mL})$ \\
\hline Penicillin & $0.12-8.0$ \\
Ampicillin & $0.12-8.0$ \\
Oxacillin & $2.0-4.0$ \\
Cephalothin & $2.0-16.0$ \\
Ceftiofur & $0.5-4.0$ \\
Penicillin + novobiocin & $1.0 / 2.0-8.0 / 16.0$ \\
Erythromycin & $0.25-4.0$ \\
Pirlimycin & $0.5-4.0$ \\
Tetracycline & $1.0-8.0$ \\
Sulfadimethoxine & $32.0-256.0$ \\
\hline
\end{tabular}




\section{Data Analysis}

Escherichia coli isolates were categorized according to the $\mathrm{CM}$ case and characteristics at the cow and herd level as follows: stage of lactation $(\leq 100,101-200$, or $\geq 201 \mathrm{~d}$ after calving); parity (primiparous or multiparous); quarter position (front or hind); CM severity (mild, changes only in the milk appearance; moderate, presence of abnormal milk accompanied by changes in the udder, or severe, combination of abnormal milk, with signs of inflammation in the udder and systemic signs; Wenz et al., 2006); season of CM occurrence in the southeastern region of Brazil (rainy: OctoberMarch; or dry: April-September; Oliveira et al., 2015); and housing system of the farm where the CM case was identified (compost-bedded pack barn, freestall, paddocks). The paddock housing system was characterized as an open area surrounded by fences or rails and without pasture for grazing.

The following logistic regression model was used to determine if descriptors at the cow level (stage of lactation, parity, quarter-position, CM severity), housing style, and season were associated with phylogenetic groups:

$$
\begin{gathered}
\text { logit }(\text { phylogroup })=\text { intercept }+ \text { housing } \\
+ \text { season }+ \text { DIM }+ \text { parity }+ \text { position }+ \text { severity } \\
+ \text { herd }(\text { random })+e,
\end{gathered}
$$

where phylogroup is the result of phylogenetic analysis (A or B1); housing (compost-bedded pack barn, freestall or paddocks); season (rainy or dry); DIM is the stage of lactation $(\leq 100,101-200$, or $\geq 201 \mathrm{~d}$ after calving); parity is related to the number of lactations (primiparous or multiparous); position is the position (front or hind) of the affected quarter; severity (mild, moderate, or severe); and $e$ is the random residual error. Herds were included in the model as random effect. Analyses were carried out using PROC GLIMMIX of SAS (version 9.4, SAS Institute Inc., Cary, NC).

Survival analysis using the PROC LIFETEST of SAS (version 9.4, SAS Institute Inc.) was completed to determine differences between AMS among E. coli phylogroups (Cortinhas et al., 2013). The concentrations of antimicrobials contained in the commercial broth microdilution test were used as the "time" variable, and the inhibition of bacterial growth was used as the event. Isolates that presented growth at the highest tested concentration were censored by the statistical model. Kaplan-Meier survival curves of the phylogenetic groups were performed for each studied antimicrobial. The null hypothesis of no differences (homogeneity of survival curves) in the survivor functions of the strata (phylogenetic groups) was evaluated using log-rank and Wilcoxon tests.

To control for the potential effect of herd-level factors (e.g., drug use practices) on the AMS of E. coli isolates, a regression analysis of survival data based on the Cox proportional hazards model was also completed to compare AMS among phylogroups using the PROC PHREG of SAS (version 9.4, SAS Institute Inc.). The shared frailty model approach was used to adjust for within-cluster (herd) correlation. The model fit was

$$
\begin{gathered}
\text { MIC } \times \text { censor }(0)=\text { phylogroup } \\
+ \text { herd }(\text { random })+e,
\end{gathered}
$$

where MIC $=$ concentration of antimicrobials contained in the commercial broth microdilution test that inhibited the bacterial growth; censor $=0$ indicated that the isolate presented bacterial growth at the highest tested concentration; phylogroup $=$ phylogenetic group to which the isolates belonged; herd (random) $=$ herd from which the isolate was identified (included in the model as random effect), and $e$ was the error term.

For all statistical analysis, significance was defined at $P \leq 0.05$.

\section{RESULTS}

\section{Phylotyping Profile and Association with Herd- and Cow-Level Descriptors}

Descriptive data of herds from which $E$. coli isolates were identified are presented in Table 2. Escherichia coli isolates were identified from cases of CM in 19 of 20 dairy herds evaluated in an epidemiological study on the characterization of CM (Tomazi, 2017). One herd (named herd $\mathrm{N}$ ) had no isolation of E. coli during the study period. The overall mean herd size was 227 $(\mathrm{SD}=333)$ lactating cows. On average, each herd was monitored for a period of 12.6 mo ( $\mathrm{SD}=1.4$; ranging from 8 to $15 \mathrm{mo}$ ). The sample collection period among farms varied according to the willingness of the owners to remain in the study.

A total of 77 cryopreserved isolates that were recultivated for this study were not selected because of the following reasons: no growth during recultivation $(\mathrm{n}=$ $26)$, MALDI score $\leq 2.0(\mathrm{n}=16)$, and contamination of the cryopreserved tube $(\mathrm{n}=8)$. In addition, other 27 isolates from 3 herds were not selected during the randomization procedure: herd A $(\mathrm{n}=13)$, herd I $(\mathrm{n}=$ $7)$, and herd $K(n=7)$. 
Therefore, 100 E. coli isolates were selected and assigned to one of the phylogroups described by Clermont et al. (2013). Most isolates belonged to phylogroups A $(\mathrm{n}=52 ; 52 \%)$ and $\mathrm{B} 1(\mathrm{n}=38 ; 38 \%)$. In addition, a total of 10 isolates (10\%) belonged to one of the other less frequent phylogenetic groups: B2 $(\mathrm{n}=2), \mathrm{C}(\mathrm{n}=$ 4), D $(\mathrm{n}=3)$, and $\mathrm{E}(\mathrm{n}=1$, Table 2$)$.

Phylogroup A was identified in 14 of 19 (73.7\%) herds, whereas group B1 was identified in $15(78.9 \%)$ herds. Phylogenetic groups identified at lower frequency were isolated from 1 (groups B2 and E), 2 (group D), and 3 herds (group C, Table 2).

Association analysis between phylogenetic groups and descriptors related to the cows and herds were performed only for the 2 most frequently identified phylogroups (A and B1). No association $(P>0.05)$ was observed in logistic regression model between phylogenetic groups and evaluated descriptors (i.e., housing type, season, stage of lactation, parity, quarter position, and CM severity; Table 3).

Association of the other identified phylogroups (B2, $\mathrm{C}, \mathrm{D}$, and $\mathrm{E}$ ) and the outcome variables were not statistically evaluated because of the low frequency of isolates $(\mathrm{n} \leq 10)$ assigned to those groups. However, the distribution of isolates within the categories of the evaluated variables is presented in Table 4.

\section{Overall Antimicrobial Susceptibility Testing}

In total, 94 isolates previously submitted for phylogenetic analysis had results of MIC (Table 5). Of isolates that did not have MIC results $(\mathrm{n}=6)$, one was contaminated (i.e., presence of $>2$ morphologically different colonies) during re-inoculation of cryopreservation tube on blood agar plate, and 5 had no growth in the positive control wells of the Sensititer panel; these isolates were excluded from the analysis.

For ampicillin, the $\mathrm{MIC}_{50}$ was $2 \mu \mathrm{g} / \mathrm{mL}$ and the $\mathrm{MIC}_{90}$ was not able to be determined, as $>10 \%$ of isolates had growth at the highest antimicrobial concentration present in the panel. The strains were highly susceptible to ceftiofur, and both $\mathrm{MIC}_{50}$ and $\mathrm{MIC}_{90}$ were $0.5 \mu \mathrm{g} /$ $\mathrm{mL}$; only one isolate was not inhibited at the highest concentration of ceftiofur. For cephalothin, the overall $\mathrm{MIC}_{50}$ was $8 \mu \mathrm{g} / \mathrm{mL}$, whereas the $\mathrm{MIC}_{90}$ was $16 \mu \mathrm{g} / \mathrm{mL}$. For sulfadimethoxine, $76.6 \%$ of isolates were susceptible at $\leq 256 \mu \mathrm{g} / \mathrm{mL}\left(\mathrm{MIC}_{50}\right)$, whereas $23.4 \%$ were not susceptible at the highest concentration contained in the

Table 2. Descriptive herd-level data, frequency of clinical mastitis (CM; overall and pathogen-specific), and frequency of phylogenetic groups of Escherichia coli isolates $(\mathrm{n}=100)$ selected from CM in 19 dairy herds of southeastern Brazil

\begin{tabular}{|c|c|c|c|c|c|c|c|c|c|c|c|c|}
\hline \multirow[b]{2}{*}{ Herd } & \multirow{2}{*}{$\begin{array}{l}\text { Lactating } \\
\text { cows }^{1}\end{array}$} & \multirow[b]{2}{*}{ Period in the study } & \multirow[b]{2}{*}{$\begin{array}{l}\text { Frequency } \\
\text { of } \mathrm{CM}^{2}\end{array}$} & \multirow[b]{2}{*}{$\begin{array}{l}\text { E. coli } \\
\mathrm{CM}^{3}\end{array}$} & \multirow{2}{*}{$\begin{array}{l}\text { Cryopreserved } \\
\text { isolates }^{4}\end{array}$} & \multirow{2}{*}{$\begin{array}{l}\text { Selected } \\
\text { isolates }^{5}\end{array}$} & \multicolumn{6}{|c|}{$\begin{array}{c}\text { Frequency of isolates } \\
\text { assigned to the phylogenetic } \\
\text { groups (no.) }\end{array}$} \\
\hline & & & & & & & $\mathrm{A}$ & B1 & B2 & $\mathrm{C}$ & $\mathrm{D}$ & $\mathrm{E}$ \\
\hline A & $1,470(52)$ & Jul. 2014-Jul. 2015 & 442 & 44 & 30 & 12 & 6 & 5 & - & 1 & - & - \\
\hline B & $184(25)$ & Apr. 2014-Apr. 2015 & 179 & 8 & 3 & 3 & - & 1 & - & 2 & - & - \\
\hline $\mathrm{C}$ & $68(5)$ & May 2014-Apr. 2015 & 36 & 3 & 3 & 3 & - & 3 & - & - & - & - \\
\hline $\mathrm{F}$ & $253(13)$ & Mar. 2014-Apr. 2015 & 874 & 17 & 9 & 6 & 5 & 1 & - & - & - & - \\
\hline G & $77(15)$ & Apr. 2014-Nov. 2014 & 43 & 3 & 2 & 2 & 1 & 1 & - & - & - & - \\
\hline $\mathrm{H}$ & $71(9)$ & Feb. 2015-Jan. 2016 & 98 & 4 & 4 & 2 & - & 2 & - & - & - & - \\
\hline I & $167(11)$ & May 2014-Apr. 2015 & 383 & 22 & 19 & 12 & 5 & 5 & - & - & 2 & - \\
\hline $\mathrm{J}$ & $120(10)$ & Apr. 2014-Apr. 2015 & 72 & 3 & 2 & 1 & 1 & - & - & - & - & - \\
\hline K & $313(7)$ & Mar. 2014-Apr. 2015 & 314 & 35 & 23 & 12 & 3 & 6 & 2 & - & - & 1 \\
\hline $\mathrm{L}$ & $194(7)$ & May 2014-Apr. 2015 & 288 & 24 & 19 & 11 & 11 & - & - & - & - & - \\
\hline S & $55(7)$ & Jun. 2014-May 2015 & 52 & 8 & 7 & 5 & 1 & 3 & - & - & 1 & - \\
\hline $\mathrm{T}$ & $46(4)$ & Apr. 2014-Apr. 2015 & 46 & 3 & 3 & 1 & - & 1 & - & - & - & - \\
\hline Overall & $227(333)$ & Mar. 2014-Jan. 2016 & 5,427 & 276 & 177 & 100 & 52 & 38 & 2 & 4 & 3 & 1 \\
\hline
\end{tabular}

${ }^{1}$ Herd size: average number of lactating cows (SD in parentheses) per herd during the study period.

${ }^{2}$ Frequency (no.) of CM cases identified during the study period regardless of the causing pathogen.

${ }^{3}$ Frequency (no.) of CM cases with identification of Escherichia coli in the bacteriological culture.

${ }^{4}$ Frequency (no.) of isolates cryopreserved during the study period.

${ }^{5}$ Frequency (no.) of isolates selected for phylogenetic analysis and antimicrobial susceptibility testing. 
Table 3. Results of a generalized linear mixed model evaluating the association between phylogenetic groups $\mathrm{A}(\mathrm{n}=52)$ and B1 $(\mathrm{n}=38)$ of Escherichia coli identified from clinical mastitis $(\mathrm{CM})$ in 19 dairy herds of southeastern Brazil and descriptors at the cow level, housing type, and season

\begin{tabular}{|c|c|c|c|c|c|c|c|}
\hline \multirow[b]{3}{*}{ Variable } & \multirow[b]{3}{*}{ Category } & \multicolumn{4}{|c|}{ Phylogenetic group } & \multirow[b]{3}{*}{$F$-value } & \multirow[b]{3}{*}{$P$-value } \\
\hline & & \multicolumn{2}{|c|}{$\mathrm{A}$} & \multicolumn{2}{|c|}{ B1 } & & \\
\hline & & No. & $\%$ & No. & $\%$ & & \\
\hline \multirow[t]{3}{*}{ Housing $(\mathrm{n}=90)$} & Freestall & 19 & 54.3 & 16 & 45.7 & \multirow[t]{3}{*}{1.06} & \multirow[t]{3}{*}{0.35} \\
\hline & $\mathrm{CBPB}^{1}$ & 8 & 44.4 & 10 & 55.6 & & \\
\hline & Paddocks $^{2}$ & 25 & 67.6 & 12 & 32.4 & & \\
\hline \multirow[t]{2}{*}{ Season $^{3}(n=90)$} & Rainy & 42 & 60.9 & 27 & 39.1 & \multirow[t]{2}{*}{3.61} & \multirow[t]{2}{*}{0.06} \\
\hline & Dry & 10 & 47.6 & 11 & 52.4 & & \\
\hline \multirow[t]{3}{*}{ Stage of lactation ${ }^{4}(\mathrm{n}=89)$} & $0-100$ & 14 & 58.3 & 10 & 41.7 & \multirow[t]{3}{*}{0.30} & \multirow[t]{3}{*}{0.74} \\
\hline & $101-200$ & 17 & 60.7 & 11 & 39.3 & & \\
\hline & $>200$ & 20 & 54.1 & 17 & 46.0 & & \\
\hline \multirow[t]{2}{*}{ Parity $(\mathrm{n}=88)$} & Primiparous & 11 & 84.6 & 2 & 15.4 & \multirow[t]{2}{*}{1.92} & \multirow[t]{2}{*}{0.16} \\
\hline & Multiparous & 40 & 53.3 & 35 & 46.7 & & \\
\hline \multirow[t]{2}{*}{ Quarter position $(\mathrm{n}=90)$} & Front & 21 & 51.2 & 20 & 48.8 & \multirow[t]{2}{*}{1.02} & \multirow[t]{2}{*}{0.32} \\
\hline & Hind & 31 & 63.3 & 18 & 36.7 & & \\
\hline \multirow{3}{*}{$\mathrm{CM}$ severity $^{5}(\mathrm{n}=85)$} & Mild & 24 & 66.7 & 12 & 33.3 & \multirow[t]{3}{*}{1.90} & \multirow[t]{3}{*}{0.16} \\
\hline & Moderate & 22 & 61.1 & 14 & 38.9 & & \\
\hline & Severe & 5 & 38.5 & 8 & 61.5 & & \\
\hline
\end{tabular}

${ }^{1} \mathrm{CBPB}=$ compost-bedded pack barn.

${ }^{2}$ The paddock housing system was characterized as an open area surrounded by fences or rails and without pasture for grazing.

${ }^{3}$ Season categories were formed by the association of the 2 characteristic seasons in southeast Brazil [rainy (October-March); or dry (April-September)]; Oliveira et al., 2015.

${ }^{4}$ Stage of lactation categorized according to DIM.

${ }^{5} \mathrm{CM}$ severity: (mild) changes only in the milk appearance; (moderate) presence of abnormal milk accompanied by changes in the udder; or (severe) combination of abnormal milk, with signs of inflammation in the udder and systemic sign.

Table 4. Distribution of less frequent phylogroups of Escherichia coli $(\mathrm{n}=10)$ identified from clinical mastitis (CM) cases in 19 dairy herds of southeastern Brazil, stratified by variables at the cow-level, housing type, and season

\begin{tabular}{|c|c|c|c|c|c|}
\hline \multirow[b]{2}{*}{ Variable } & \multirow[b]{2}{*}{ Categories } & \multicolumn{4}{|c|}{ Phylogenetic group } \\
\hline & & B2 & $\mathrm{C}$ & $\mathrm{D}$ & $\mathrm{E}$ \\
\hline \multirow[t]{3}{*}{ Housing $(\mathrm{n}=10)$} & Freestall & 2 & 1 & - & 1 \\
\hline & $\mathrm{CBPB}^{1}$ & - & 3 & 2 & - \\
\hline & Paddocks ${ }^{2}$ & - & - & 1 & - \\
\hline \multirow[t]{2}{*}{$\operatorname{Season}^{3}(n=10)$} & Rainy & 2 & 4 & 3 & 1 \\
\hline & Dry & - & - & - & - \\
\hline \multirow[t]{3}{*}{ Stage of lactation ${ }^{4}(\mathrm{n}=9)$} & $0-100$ & - & 1 & 1 & - \\
\hline & $101-200$ & - & 2 & 1 & - \\
\hline & $>200$ & 2 & 1 & 1 & - \\
\hline \multirow[t]{2}{*}{ Parity $(\mathrm{n}=9)$} & Primiparous & - & 2 & 1 & - \\
\hline & Multiparous & 2 & 2 & 2 & - \\
\hline \multirow[t]{2}{*}{ Quarter position $(\mathrm{n}=10)$} & Front & 2 & - & - & 1 \\
\hline & Hind & - & 4 & 3 & - \\
\hline \multirow[t]{3}{*}{$\mathrm{CM}_{\text {severity }}{ }^{5}(\mathrm{n}=9)$} & Mild & 1 & 1 & - & - \\
\hline & Moderate & - & 2 & 3 & 1 \\
\hline & Severe & 1 & - & - & - \\
\hline
\end{tabular}

${ }^{1} \mathrm{CBPB}=$ compost-bedded pack barn.

${ }^{2}$ The paddock housing system was characterized as an open area surrounded by fences or rails and without pasture for grazing.

${ }^{3}$ Season categories were formed by the association of the 2 characteristic seasons in southeast Brazil [rainy (October-March); or dry (April-September)]; Oliveira et al., 2015.

${ }^{4}$ Stage of lactation categorized according to days in milk.

${ }^{5} \mathrm{CM}$ severity: (mild) changes only in the milk appearance, (moderate) presence of abnormal milk accompanied by changes in the udder; or (severe) combination of abnormal milk, with signs of inflammation in the udder and systemic sign. 
Table 5. Overall frequency $(\%)$ of Escherichia coli isolates $(\mathrm{n}=94)$ that had $50 \%\left(\mathrm{MIC}_{50}\right)$ and $90 \%(\mathrm{MIC}$ ) of bacterial growth inhibited at each antimicrobial concentration

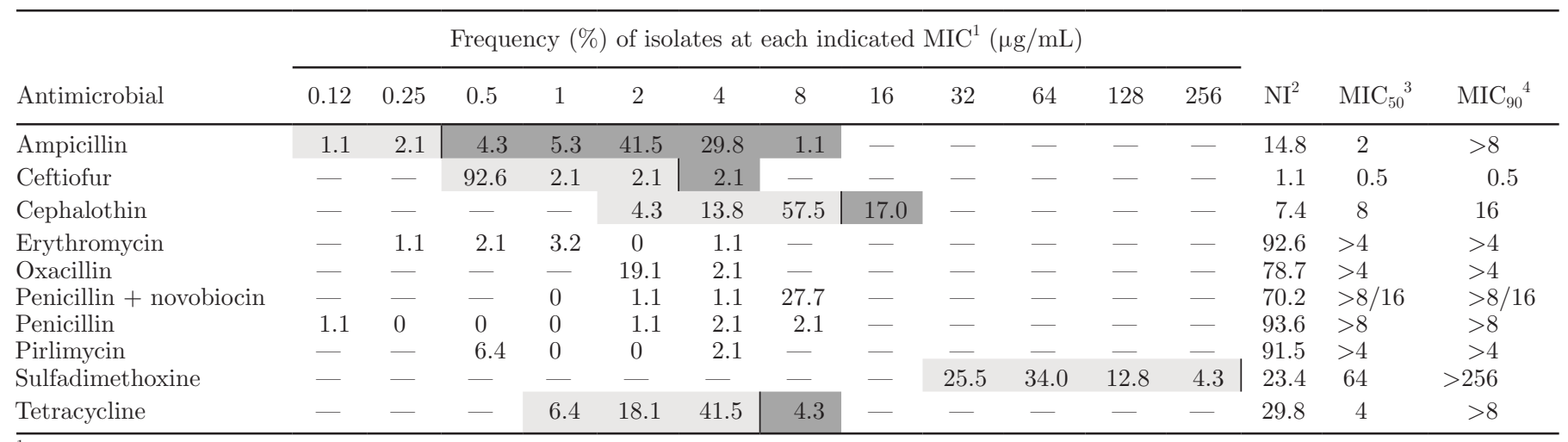

${ }^{1}$ The light gray shading represents the susceptible zone, and the darker gray shading represents the resistant zone. A dash (-) indicates that the antimicrobial concentration was not tested. Results were interpreted according to the CLSI (2013). Interpretative criteria were based on human data (cephalothin, sulfadimethoxine, and tetracycline), dog data (ampicillin), and bovine mastitis (ceftiofur). The resistant category included those isolates categorized as either intermediate or resistant. Antimicrobials without shading have no interpretive criteria available for Escherichia coli.

${ }^{2} \mathrm{NI}=$ not inhibited (growth at highest concentration tested).

${ }^{3} \mathrm{MIC}(\mu \mathrm{g} / \mathrm{mL})$ that inhibited $50 \%\left(\mathrm{MIC}_{50}\right)$ of the isolates.

${ }^{4} \mathrm{MIC}(\mu \mathrm{g} / \mathrm{mL})$ that inhibited $90 \%\left(\mathrm{MIC}_{90}\right)$ of the isolates.

test. The overall $\mathrm{MIC}_{50}$ for tetracycline was $4 \mu \mathrm{g} / \mathrm{mL}$, whereas $29.8 \%$ of the isolates were not inhibited at the highest concentration present in the test.

For erythromycin, oxacillin, penicillin, penicillin/novobiocin, and pirlimycin, more than $70 \%$ of strains were not inhibited at the highest antimicrobial concentration contained in the panel (Table 5).

\section{Antimicrobial Susceptibility Testing of Phylogenetic Groups A and B1}

The frequency distribution of $\mathrm{MIC}$ results $\left(\mathrm{MIC}_{50}\right.$ and $\mathrm{MIC}_{90}$ ), according to the phylogenetic groups $\mathrm{A}$ and $\mathrm{B} 1$ of $E$. coli isolates, are shown in Table 6 . The $\mathrm{MIC}_{50}$ of ampicillin for E. coli isolates of both phylogroups (A and B1) was $2 \mu \mathrm{g} / \mathrm{mL}$. The $\mathrm{MIC}_{90}$ for the same antimicrobial was $4 \mu \mathrm{g} / \mathrm{mL}$ for isolates of phylogroup $\mathrm{A}$; however, the $\mathrm{MIC}_{90}$ for isolates of phylogroup B1 was not determined because more than $10 \%$ of tested isolates were not inhibited at the highest concentration of ampicillin present in the panel. In total, $10 \%$ of isolates assigned to phylogroup A, and $14 \%$ of isolates of phylogroup B1 were not inhibited at the highest concentration of ampicillin. Only $2 \%$ of isolates from phylogroup A and 5.8\% from the phylogroup B1 were susceptible to ampicillin.

For ceftiofur, the $\mathrm{MIC}_{50}$ and $\mathrm{MIC}_{90}$ was observed at the lowest concentration $(0.5 \mu \mathrm{g} / \mathrm{mL})$ for both phylogenetic groups (A and B1). Only 2\% (1 of 50) of isolates from phylogroup $\mathrm{A}$ and $5.8 \%$ (2 of 34 ) from phylogroup B1 were not susceptible to ceftiofur. The $\mathrm{MIC}_{50}$ of cephalothin $(8 \mu \mathrm{g} / \mathrm{mL})$ was also similar among phylogroups $\mathrm{A}$ and $\mathrm{B} 1$; however, the $\mathrm{MIC}_{90}$ was $16 \mu \mathrm{g} / \mathrm{mL}$ for isolates assigned to phylogroup $\mathrm{A}$ and $>16 \mu \mathrm{g} / \mathrm{mL}$ for isolates assigned to phylogroup B1. A total of $86 \%$ of isolates belonging to phylogroup A and $61.8 \%$ of isolates from phylogroup B1 were susceptible to cephalothin (Table 6).

The MIC of sulfadimethoxine to inhibit $50 \%$ of isolates from both phylogroups A and B1 was $64 \mu \mathrm{g} / \mathrm{mL}$. On the other hand, estimation of $\mathrm{MIC}_{90}$ for sulfadimethoxine was not possible because more than $10 \%$ of tested isolates were resistant to this antimicrobial at the highest concentration present in the commercial panel. A total of $80 \%$ of isolates assigned to phylogroup A and $76.5 \%$ of isolates of phylogroup B1 were susceptible to sulfadimethoxine.

A total of $66 \%$ of isolates from phylogroup A and $67.6 \%$ from phylogroup B1 were susceptible to tetracycline. For this same antimicrobial, the $\mathrm{MIC}_{50}$ was similar for both groups $(4 \mu \mathrm{g} / \mathrm{mL})$, whereas the $\mathrm{MIC}_{90}$ was greater than the highest antimicrobial concentration $(8 \mu \mathrm{g} / \mathrm{mL})$.

For antimicrobials with no expected antimicrobial activity against $E$. coli, the isolates of both phylogroups $\mathrm{A}$ and $\mathrm{B} 1$ were resistant to the highest antimicrobial concentration contained in the panel in more than $70 \%$ of the tests for oxacillin and penicillin-novobiocin, and in more than $90 \%$ for erythromycin, penicillin, and pirlimycin. 


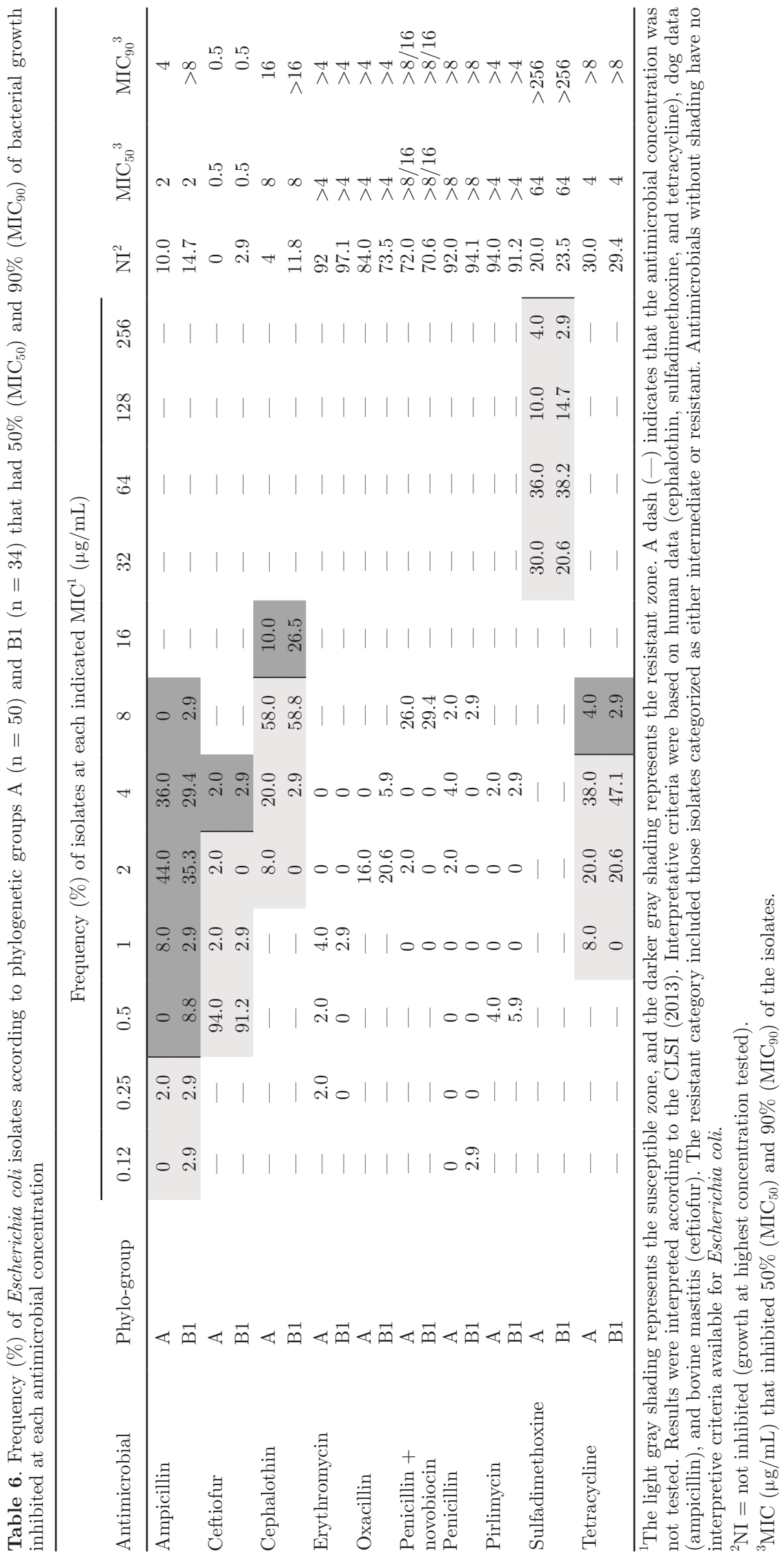


Table 7. Results of survival function analysis and Cox proportional hazards model comparing the antimicrobial susceptibility of Escherichia coli isolated from clinical mastitis cases identified in 19 dairy herds and stratified by the phylogeny groups A $(\mathrm{n}=50)$ and B1 (n = 34)

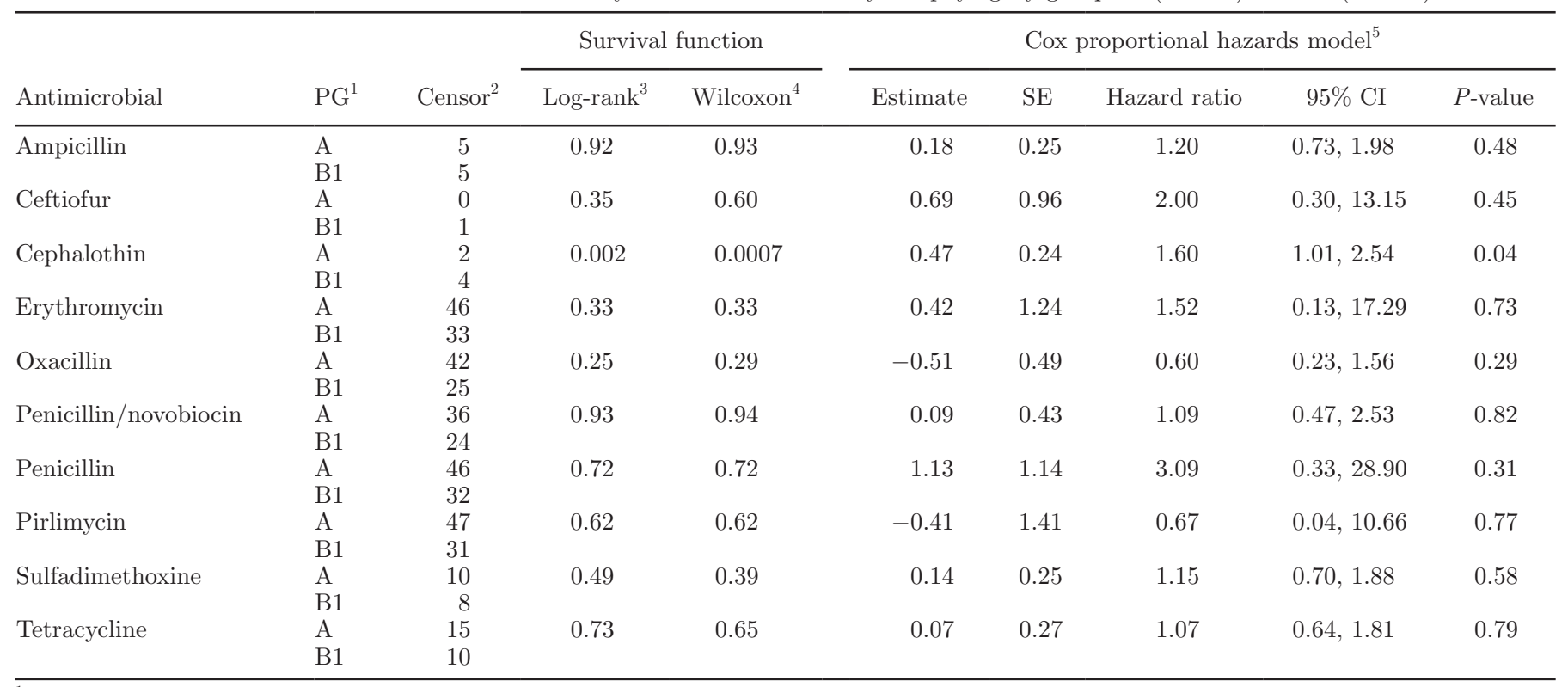

${ }^{1} \mathrm{PG}=$ phylogenetic group.

${ }^{2}$ Frequency of isolates censored (observed growth at the highest antimicrobial drug concentration of the used test).

${ }^{3}$ Log-rank test for equality of strata at higher antimicrobial concentrations.

${ }^{4}$ Wilcoxon test for equality of strata at lower antimicrobial concentrations.

${ }^{5}$ Herd was included in the model as a random effect.

\section{Survival Function Analysis}

Antimicrobial susceptibility among phylogenetic groups A and B1 were compared using Kaplan-Meier survival curves and Cox proportional hazards model (Table 7). Homogeneous survival curves were observed for all evaluated antimicrobials (log-rank test $>0.25$; Wilcoxon >0.29), except for cephalothin (log-rank test $=0.002 ;$ Wilcoxon $=0.0007)$. Survival curves showed heterogeneity among phylogenetic groups for cephalothin, indicating that isolates of phylogroup A were inhibited at lower concentration of this antimicrobial than isolates of phylogroup B1 (Figure 1). Similarly, cephalothin was the only antimicrobial showing difference in AMS among phylogroups A and B1 when the Cox proportional hazards model was used (Table 7).

\section{DISCUSSION}

To our knowledge, this is the first study evaluating the association of phylogenetic groups and AMS of $E$. coli isolated from bovine $\mathrm{CM}$ in Brazil. Phylogenetic studies may provide insights into the epidemiology of E. coli associated with bovine mastitis and may indicate specific host-pathogen relationships (Tenaillon et al., 2010). However, our data showed no association between E. coli phylogenetic groups and cow-level de- scriptors, housing system, or CM severity. Furthermore, differences in AMS may be uncommon as they were limited to only one antimicrobial (i.e., cephalothin). Nevertheless, our results on AMS of E. coli causing

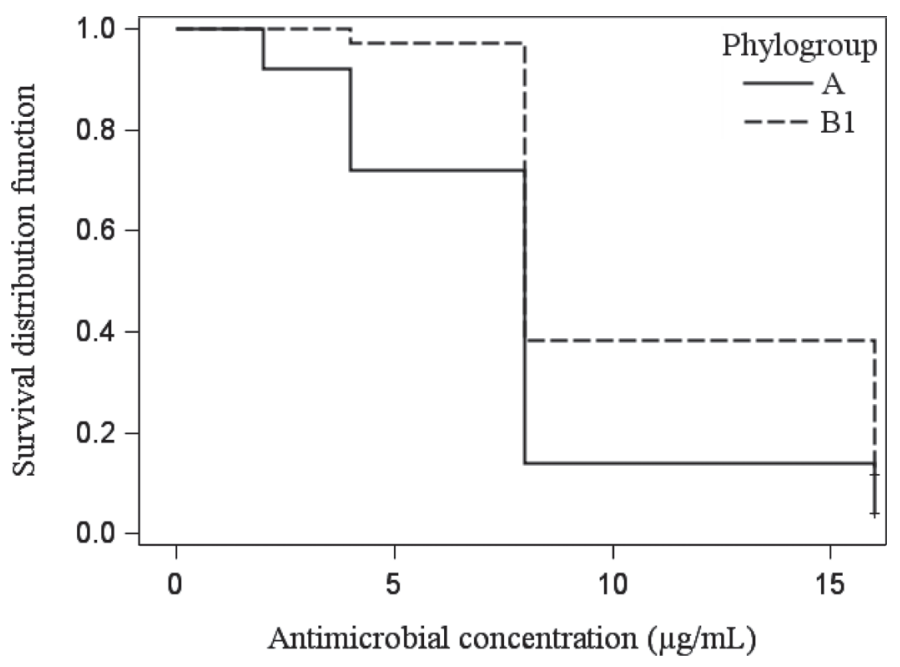

Figure 1. Heterogeneous Kaplan-Meier survival curves (log-rank test $=0.002$; Wilcoxon test $=0.0007)$ for Escherichia coli isolated from clinical mastitis in 19 dairy herds, according to cephalothin susceptibility testing and stratified by phylogenetic groups A $(\mathrm{n}=50)$ or B1 $(\mathrm{n}=34)$. Censored data are indicated as a cross on the right end of the lines. 
mastitis can be used as a basis for further pharmacoepidemiological studies to monitor the emergence of potential resistant strains.

In our study, $90 \%$ of $E$. coli isolates were assigned to phylogroups A (58\%) or B1 (32\%), and a small proportion $(10 \%)$ of isolates were assigned to phylogroups B2 $(2.0 \%), \mathrm{C}(4.0 \%), \mathrm{D}(3.0 \%)$, and $\mathrm{E}(1.0 \%)$, whereas no isolates were identified as belonging to group F. High frequency of phylogroups A and B1 were also observed in other studies evaluating the phylogeny of E. coli isolated from CM (Liu et al., 2014; Keane, 2016; Zhang et al., 2018). In a recent study conducted in China, of 79 E. coli isolates from bovine mastitis, $59.5 \%$ belonged to phylogenetic group A, whereas $27.8 \%$ were assigned to phylogroup B1 (Zhang et al., 2018). Similarly, in a study conducted in Ireland, 54\% (20/37) of E. coli isolates recovered from $\mathrm{CM}$ were assigned to phylogroup A and 32\% (12/37) to group B1 (Keane, 2016). Phylogenetic groups A and B1 were mainly associated with commensal strains of $E$. coli in dairy cattle (Son et al., 2009), which may explain the highest prevalence (>85\%) of these groups among isolates recovered from bovine IMI in our study and other studies.

Goldstone et al. (2016) described that E. coli assigned to phylogroup A (the most common phylogroup causing mastitis) have some unique characteristics, such as genomic content and phylogenetic similarity, indicating that some functional products of the genes may be essential for E. coli causing IMI. Moreover, E. coli associated with mastitis still cannot be distinguished from bovine commensal $E$. coli based on the presence of virulence-associated factors, such as is performed for intestinal pathogenic E. coli. However, it is known that the presence of few putative genes in mastitis-associated $E$. coli are important for gastrointestinal colonization (Leimbach et al., 2017). Therefore, because E. coli causing mastitis are mostly associated with environmental sources, the control of this pathogen should focus on management and hygiene practices aiming to reduce exposure of teat ends to intestinal pathogenic strains. A study comparing phylotypes among fecal commensal $E$. coli isolated on the same farms (and time) where the isolates from $\mathrm{CM}$ were obtained could improve epidemiological understanding of E. coli phylogroups causing mastitis.

Although there was high prevalence of isolates belonging to phylogroups $\mathrm{A}$ and $\mathrm{B} 1$, other less frequently isolated phylogroups (B2, C, D, and E) were also identified in our study, which indicates their potential to cause CM in dairy cows. Phylogroups B2 and D were also described in other reports evaluating $E$. coli isolated from IMI. Isolates belonging to group B2 had also low frequency $(1.4 \%$; 2/144 isolates) in a Finnish study evaluating isolates recovered from CM (Suojala et al.,
$2011)$, and in a Chinese study $(4.3 \% ; 2 / 46)$ evaluating multi-drug-resistant extended-spectrum $\beta$-lactamaseproducing $E$. coli isolated from bovine mastitis (Ali et al., 2017). Similarly, another study found that only 2 of 30 E. coli isolates pertained to phylogroup B2 (Dogan et al., 2012). On the other hand, phylogroup D was reported in higher frequency in studies about mastitis, described in $11.1 \%$ (second most frequent phylogroup) of isolates in the study of Suojala et al. (2011); in 16.4\% of isolates recovered from CM in dairy herds of Iran (Ghanbarpour and Oswald, 2010); in 14\% of isolates in an Irish study (Keane, 2016), and in $21.7 \%$ of extendedspectrum $\beta$-lactamase-producing $E$. coli in the study conducted in China (Ali et al., 2017).

Some phylogroups identified in our study were not described (e.g., phylogroup C), or had low frequency in other studies evaluating their relationship with IMI in dairy cows (e.g., phylogroup E; Kempf et al., 2016). Because many phylogenetic studies were based on the initial rapid determination scheme purposed by Clermont et al. (2000), which only permits distinguishing isolates among 4 phylogenetic groups (A, B1, B2, and $\mathrm{D})$, the frequency of groups $\mathrm{C}$ and $\mathrm{E}$ may have been underestimated in previous studies. Using the quadruplex PCR method, it is possible to assign E. coli into 8 phylogroups: 7 belonging to $E$. coli sensu stricto (A, B1, B2, C, D, E, F), whereas the eighth is the Escherichia cryptic clade I (Clermont et al., 2013).

Previous studies have shown that mastitis-associated strains were found across 5 (A, B1, B2, D, and E) of 7 E. coli sensu stricto groups (Kempf et al., 2016). However, to the best of our knowledge, this is the first study reporting E. coli of phylogroup $\mathrm{C}$ as the cause of CM. In other studies, strains belonging to phylogroup $\mathrm{C}$ were associated with intestinal and extra-intestinal infections in humans (Clermont et al., 2011), and with fecal samples collected from diarrheal calves (Souto et al., 2017). Only 4 isolates were assigned to phylogroup C in our study and few isolates were assigned to phylogroups B2, D, and E. Therefore, further studies with a larger number of strains could better clarify whether these less frequently identified phylogroups are only opportunistic pathogens or are emerging phylogroups associated with CM in dairy cows.

None of the factors or descriptors evaluated in our study (i.e., housing system, season, quarter position, stage of lactation, parity, CM severity) were associated with the phylogenetic groups. Therefore, our results did not allow us to reject the null hypothesis that phylogroup (A or B1) is independent of evaluated variables. As both phylogroups A and B1 were reported to have specific virulence factors (Liu et al., 2014), it could be speculated that differences in mastitis severity could be attributed to the phylogenetic group; how- 
ever, no differences of CM severity were found among the phylogroups in our study. Likewise, another study found no association among phylogenetic groups with clinical signs of dairy cows with acute CM (Suojala et al., 2011). Furthermore, a review on E. coli CM reported that clinical signs are mainly determined by cow factors such as immunological status or hormonal and metabolic alterations, rather than specific features of the bacterial strain (Burvenich et al., 2003). Conversely, immune response and mammary pathogenesis can vary according to E. coli strains causing IMI. Differences in the intensity and duration of cellular (e.g., SCC) and other mammary immune responses (e.g., type of cytokine secreted) was observed in a challenge study comparing 3 distinct MPEC isolated from 3 forms of mastitis (peracute, persistent, and acute; Blum et al., 2017).

Escherichia coli isolates in our study were submitted for analysis of AMS for determination of $\mathrm{MIC}_{50}$ and $\mathrm{MIC}_{90}$, and a high frequency of isolates were considered resistant to most of antimicrobials tested. Overall, more than $96 \%$ of strains were resistant to ampicillin, and more than $23 \%$ were considered resistant to cephalothin, sulfadimethoxine, or tetracycline. It is important to mention that a direct comparison between studies cannot be easily performed, because several factors can affect the susceptibility results, such as methodology used for susceptibility testing (disk diffusion vs. broth dilution method), concentration of antimicrobials used in MIC analysis, and differences in the interpretive criteria used for categorizing isolates as susceptible or resistant.

Only 2 reports were found describing the same commercial panel as used in our study for AMS testing of E. coli strains (Rajala-Schultz et al., 2004; Hoe and Ruegg, 2005). In comparison to our study, RajalaSchultz et al. (2004) reported lower frequency of resistance to ampicillin (44.4 vs. $96.8 \%$ in our study), but higher resistance frequency to the rest of the antimicrobials tested. Although a higher resistance to ampicillin was observed in our study, the $\mathrm{MIC}_{50}$ was lower $(2 \mu \mathrm{g} /$ $\mathrm{mL})$ in comparison to the aforementioned report $(4 \mu \mathrm{g} /$ $\mathrm{mL}$ ). Lower $\mathrm{MIC}_{50}$ were also observed in our study for sulfadimethoxine ( $64 \mathrm{vs} .>256 \mu \mathrm{g} / \mathrm{mL}$ ) and tetracycline ( 4 vs. $8 \mu \mathrm{g} / \mathrm{mL}$ ) in comparison to the results found by Rajala-Schultz et al. (2004), and similar $\mathrm{MIC}_{50}$ were observed between studies for ceftiofur $(0.5 \mu \mathrm{g} / \mathrm{mL})$ and cephalothin $(8 \mu \mathrm{g} / \mathrm{mL})$. Furthermore, our results showed lower $\mathrm{MIC}_{90}$ for ceftiofur $(0.5 \mu \mathrm{g} / \mathrm{mL})$ and cephalothin $(16 \mu \mathrm{g} / \mathrm{mL})$ than observed in the study of Rajala-Schultz et al. (2004), in which the $\mathrm{MIC}_{90}$ was 2.0 $\mu \mathrm{g} / \mathrm{mL}$ for ceftiofur and $>16 \mu \mathrm{g} / \mathrm{mL}$ for cephalothin.

Hoe and Ruegg (2005) evaluated in vitro AMS of E. coli isolated from cows with $\mathrm{CM}$ and reported an overall susceptibility of $E$. coli similar to our results. However, $91 \%$ of isolates were reported as susceptible to ampicillin in the study, whereas only $3.2 \%$ of isolates was susceptible to this antimicrobial in our study. The difference in MIC results for ampicillin between studies is due to different interpretive criteria for classification of the isolates as susceptible or resistant. We used 0.25 $\mu \mathrm{g} / \mathrm{mL}$ as cut-off (criteria used for dog's skin and soft tissue), while a higher limit $(8.0 \mu \mathrm{g} / \mathrm{mL})$ was used in the study of Hoe and Ruegg (2005), which was based on human criteria. Adopting the same interpretative criteria as used by Hoe and Ruegg (2005), 85.1\% (80/94) of $E$. coli isolates would be considered susceptible to ampicillin in our study.

High proportions of antimicrobial resistance $(>70 \%)$ were also observed for erythromycin, oxacillin, penicillin, penicillin associated with novobiocin, and pirlimycin. However, these antimicrobials are not expected to have activity against gram-negative bacteria, and no interpretive criteria are available for susceptibility analysis of E. coli strains. Similar outcomes were observed in other studies (Rajala-Schultz et al., 2004; Hoe and Ruegg, 2005). Future studies evaluating AMS of mastitis-causing E. coli may consider using panels with antimicrobials that have a more specific spectrum of activity against gram-negative organisms. Nevertheless, our results about AMS are still relevant considering global concerns about the rational use of antimicrobials. Blanket protocols (instead of culture based) are still widely used for CM treatment in dairy herds; a high frequency of E. coli $\mathrm{CM}$ cases have been treated with antimicrobials included in the panel evaluated in this study, or with other formulations with greater activity against gram-positive organisms.

Most strains (96.8\%) were susceptible to ceftiofur and comparable results were reported in other studies. Bengtsson et al. (2009) reported that ceftiofur at a concentration of $0.5 \mu \mathrm{g} / \mathrm{mL}$ inhibited $96.3 \%$ of 163 E. coli strains isolated from acute CM in dairy cows. In another study, $100 \%$ of $E$. coli strains isolated from $\mathrm{CM}$ were susceptible to ceftiofur at a concentration of $0.5 \mu \mathrm{g} / \mathrm{mL}$ (Thomas et al., 2015). Ceftiofur is a third-generation cephalosporin that acts by inhibiting bacterial cell wall synthesis of both gram-negative and gram-positive bacteria (Schukken et al., 2011). Because this antimicrobial was marketed to be administered via the intramammary route, it has been used extensively for CM treatment in dairy cattle (Saini et al., 2012; Oliveira and Ruegg, 2014).

Kaplan-Meier curves and survival analysis were used in our study to determine whether phylogroups A and B1 had different MIC for evaluated antimicrobials. In general, it is assumed in survival analysis that events may occur over a continuous scale, such as time (Cor- 
tinhas et al., 2013). In our study, we assumed that MIC determined by broth microdilution was the continuous variable, which allowed us to compare the shape of survival curves of the susceptibility test. An important feature of this method is the possibility of inclusion in the analysis of those isolates that were not inhibited at the highest concentration. On the other hand, a drawback of this method is the inability to analyze survival data with clustered events. In our study, herd-level factors (e.g., overuse of certain antimicrobials) could affect AMS of E. coli isolates. Therefore, not considering the herd effect in the data analysis may bias the outcomes by violation of the assumption of independence of the observations. Hence, in addition to the estimation of the Kaplan-Meier curves, we performed a regression analysis of survival data based on the Cox proportional hazards model. This method allowed us to compare AMS between phylogroups A and B1 by controlling the potential effect of herds on the outcomes.

In both survival methods, a difference in AMS among phylogroups was observed only for cephalothin, in which strains belonging to phylogroup A were inhibited at lower antimicrobial concentrations than strains assigned to phylogroup B1. For the remaining antimicrobials, the curves comparing AMS were homogeneous between phylogroups A and B1. Studies evaluating the relationship of phylogenetic groups and susceptibility to antimicrobials are scant in the indexed literature (Liu et al., 2014; Keane, 2016). Furthermore, studies using survival analysis to compare the susceptibility of antimicrobials among phylogenetic groups were not found, which makes it difficult to compare our results with those in other studies.

A recent study conducted in Ireland also reported a high prevalence of strains belonging to phylogroups A and B1, although no relationship between antimicrobial resistance and phylogenetic group was observed. In another study, $70 \mathrm{E}$. coli isolates recovered from clinical and subclinical mastitis were characterized with respect to their phylogenetic groups, virulence factors, and AMS (Liu et al., 2014). The results of this study showed that $67.1 \%$ of isolates were resistant to streptomycin, and those from group B1 were more resistant to this antimicrobial than isolates from group A. Isolates belonging to phylogroup B1 were also reported to have a higher frequency of virulence genes than isolates of group A, which could be related to potential differences in antimicrobial resistance among phylogroups.

Despite these promising results, we recognized that a lower sampling size than expected was used for evaluation of descriptors at the cow or herd level and phylogenetic groups, especially for less frequent phylogroups. In addition, further studies evaluating AMS and the presence of resistance genes for these less frequently identified phylogroups are needed to better understand their epidemiology and potential role in the etiology of mastitis. Despite these limitations, this is the first study evaluating the phylogeny and susceptibility of $E$. coli strains isolated from CM in dairy herds in Brazil. The results observed in this report may be used as a reference for further studies evaluating the pharmacoepidemiology and pathogenicity of E. coli causing CM in dairy cows.

In conclusion, E. coli isolates associated with CM mainly belonged to phylogenetic groups A and B1, although isolates were also assigned at lower frequency to phylogenetic groups B2, C, D, and E. None of the cowand herd-level descriptors were associated with the $E$. coli phylogenetic groups. Resistance was observed for all evaluated antimicrobials. However, E. coli isolates were more susceptible to antimicrobials pertaining to the cephalosporin class. Finally, strains belonging to group A1 were inhibited at lower cephalothin concentration than strains assigned to phylogroup B1, which indicates that specific genetic properties among certain strains may be involved with cephalothin susceptibility. Based on our results, management strategies to reduce exposure of cows' teat ends to commensal $E$. coli (regardless of phylogenetic group) are still the best practice for CM control in dairy herds. In addition, prudent antimicrobial use should be performed to treat CM caused by E. coli, as phylogenetic properties may affect treatment outcomes.

\section{ACKNOWLEDGMENTS}

We are grateful to Fundação de Amparo à Pesquisa do Estado de São Paulo (FAPESP, Brazil; grant numbers 2013/22286-3, 2014/17411-6, and 2015/103326) and to Conselho Nacional de Desenvolvimento Científico e Tecnológico (CNPq, Brazil; grant number 448357/2014-3) for financial support. Our thanks also go to Bruna Gomes Alves, Gustavo Freu, and Carlos Fidelis (Qualileite Milk Quality Laboratory, School of Veterinary Medicine and Animal Science, University of São Paulo, Pirassununga, Brazil) for their collaboration with laboratory analysis.

\section{REFERENCES}

Ali, T., S. U. Rahman, L. Zhang, M. Shahid, D. Han, J. Gao, S. Zhang, P. L. Ruegg, U. Saddique, and B. Han. 2017. Characteristics and genetic diversity of multi-drug resistant extended-spectrum betalactamase (ESBL)-producing Escherichia coli isolated from bovine mastitis. Oncotarget 8:90144-90163.

Barreiro, J. R., C. R. Ferreira, G. B. Sanvido, M. Kostrzewa, T. Maier, B. Wegemann, V. Böttcher, M. N. Eberlin, and M. V. dos Santos. 2010. Short communication: Identification of subclinical cow mastitis pathogens in milk by matrix-assisted laser desorption/ionization time-of-flight mass spectrometry. J. Dairy Sci. 93:5661-5667. 
Bengtsson, B., H. E. Unnerstad, T. Ekman, K. Artursson, M. NilssonOst, and K. P. Waller. 2009. Antimicrobial susceptibility of udder pathogens from cases of acute clinical mastitis in dairy cows. Vet. Microbiol. 136:142-149.

Blum, S. E., E. D. Heller, S. Jacoby, O. Krifucks, and G. Leitner. 2017. Comparison of the immune responses associated with experimental bovine mastitis caused by different strains of Escherichia coli. J. Dairy Res. 84:190-197.

Burvenich, C., V. Van Merris, J. Mehrzad, A. Diez-Fraile, and L. Duchateau. 2003. Severity of E. coli mastitis is mainly determined by cow factors. Vet. Res. 34:521-564.

Clermont, O., S. Bonacorsi, and E. Bingen. 2000. Rapid and simple determination of the Escherichia coli phylogenetic group. Appl. Environ. Microbiol. 66:4555-4558.

Clermont, O., J. K. Christenson, E. Denamur, and D. M. Gordon. 2013. The Clermont Escherichia coli phylo-typing method revisited: Improvement of specificity and detection of new phylo-groups. Environ. Microbiol. Rep. 5:58-65.

Clermont, O., M. Olier, C. Hoede, L. Diancourt, S. Brisse, M. Keroudean, J. Glodt, B. Picard, E. Oswald, and E. Denamur. 2011. Animal and human pathogenic Escherichia coli strains share common genetic backgrounds. Infect. Genet. Evol. 11:654-662.

Clinical and Laboratory Standards Institute (CLSI). 2013. Performance Standards for Antimicrobial Disk and Dilution Susceptibility Tests for Bacteria Isolated From Animals; Approved Standard, 4th ed. Clinical and Laboratory Standards Institute, Wayne, PA.

Cortinhas, C. S., L. Oliveira, C. A. Hulland, M. V. Santos, and P. L. Ruegg. 2013. Minimum inhibitory concentrations of cephalosporin compounds and their active metabolites for selected mastitis pathogens. Am. J. Vet. Res. 74:683-690.

Dogan, B., M. Rishniw, G. Bruant, J. Harel, Y. H. Schukken, and K. W. Simpson. 2012. Phylogroup and lpfA influence epithelial invasion by mastitis associated Escherichia coli. Vet. Microbiol. 159:163-170.

Dohoo, I. R., J. Smith, S. Andersen, D. F. Kelton, and S. Godden. and Mastitis Research Workers' Conference. 2011. Diagnosing intramammary infections: Evaluation of definitions based on a single milk sample. J. Dairy Sci. 94:250-261.

Fan, H. H., S. H. Kleven, and M. W. Jackwood. 1995. Application of polymerase chain reaction with arbitrary primers to strain identification of Mycoplasma gallisepticum. Avian Dis. 39:729-735.

Ghanbarpour, R., and E. Oswald. 2010. Phylogenetic distribution of virulence genes in Escherichia coli isolated from bovine mastitis in Iran. Res. Vet. Sci. 88:6-10.

Goldstone, R. J., S. Harris, and D. G. Smith. 2016. Genomic content typifying a prevalent clade of bovine mastitis-associated Escherichia coli. Sci. Rep. 6:30115.

Hoe, F. G., and P. L. Ruegg. 2005. Relationship between antimicrobial susceptibility of clinical mastitis pathogens and treatment outcome in cows. J. Am. Vet. Med. Assoc. 227:1461-1468.

Keane, O. M. 2016. Genetic diversity, the virulence gene profile and antimicrobial resistance of clinical mastitis-associated Escherichia coli. Res. Microbiol. 167:678-684.

Kempf, F., C. Slugocki, S. E. Blum, G. Leitner, and P. Germon. 2016. Genomic comparative study of bovine mastitis Escherichia coli. PLoS One 11:e0147954.

Klaas, I. C., and R. N. Zadoks. 2017. An update on environmental mastitis: Challenging perceptions. Transbound. Emerg. Dis. https: //doi.org/10.1111/tbed.12704.

Lago, A., S. M. Godden, R. Bey, P. L. Ruegg, and K. Leslie. 2011. The selective treatment of clinical mastitis based on on-farm culture results: I. Effects on antibiotic use, milk withholding time, and short-term clinical and bacteriological outcomes. J. Dairy Sci. 94:4441-4456.

Leimbach, A., A. Poehlein, J. Vollmers, D. Görlich, R. Daniel, and U. Dobrindt. 2017. No evidence for a bovine mastitis Escherichia coli pathotype. BMC Genomics 18:359.
Liu, Y., G. Liu, W. Liu, Y. Liu, T. Ali, W. Chen, J. Yin, and B. Han. 2014. Phylogenetic group, virulence factors and antimicrobial resistance of Escherichia coli associated with bovine mastitis. Res. Microbiol. 165:273-277.

National Mastitis Council. 1999. Laboratory Handbook on Bovine Mastitis. National Mastitis Council, Verona, WI.

Oliveira, C. S., H. Hogeveen, A. M. Botelho, P. V. Maia, S. G. Coelho, and J. P. Haddad. 2015. Cow-specific risk factors for clinical mastitis in Brazilian dairy cattle. Prev. Vet. Med. 121:297-305.

Oliveira, L., C. Hulland, and P. L. Ruegg. 2013. Characterization of clinical mastitis occurring in cows on 50 large dairy herds in Wisconsin. J. Dairy Sci. 96:7538-7549.

Oliveira, L., and P. L. Ruegg. 2014. Treatments of clinical mastitis occurring in cows on 51 large dairy herds in Wisconsin. J. Dairy Sci. 97:5426-5436.

Rajala-Schultz, P. J., K. L. Smith, J. S. Hogan, and B. C. Love. 2004 Antimicrobial susceptibility of mastitis pathogens from first lactation and older cows. Vet. Microbiol. 102:33-42.

Saini, V., J. T. McClure, D. Léger, S. Dufour, A. G. Sheldon, D. T. Scholl, and H. W. Barkema. 2012. Antimicrobial use on Canadian dairy farms. J. Dairy Sci. 95:1209-1221.

Schukken, Y. H., G. J. Bennett, M. J. Zurakowski, H. L. Sharkey, B. J. Rauch, M. J. Thomas, B. Ceglowski, R. L. Saltman, N. Belomestnykh, and R. N. Zadoks. 2011. Randomized clinical trial to evaluate the efficacy of a 5-day ceftiofur hydrochloride intramammary treatment on nonsevere gram-negative clinical mastitis. J. Dairy Sci. 94:6203-6215.

Shpigel, N. Y., S. Elazar, and I. Rosenshine. 2008. Mammary pathogenic Escherichia coli. Curr. Opin. Microbiol. 11:60-65.

Son, I., J. A. Van Kessel, and J. S. Karns. 2009. Genotypic diversity of Escherichia coli in a dairy farm. Foodborne Pathog. Dis. 6:837-847.

Souto, M. S., F. M. Coura, E. M. Dorneles, A. P. Stynen, T. M. Alves, J. A. Santana, R. B. Pauletti, R. M. Guedes, A. M. Viott, M. B. Heinemann, and A. P. Lage. 2017. Antimicrobial susceptibility and phylotyping profile of pathogenic Escherichia coli and Salmonella enterica isolates from calves and pigs in Minas Gerais, Brazil. Trop. Anim. Health Prod. 49:13-23.

Suojala, L., L. Kaartinen, and S. Pyorala. 2013. Treatment for bovine Escherichia coli mastitis-An evidence-based approach. J. Vet. Pharmacol. Ther. 36:521-531.

Suojala, L., T. Pohjanvirta, H. Simojoki, A. L. Myllyniemi, A. Pitkala, S. Pelkonen, and S. Pyorala. 2011. Phylogeny, virulence factors and antimicrobial susceptibility of Escherichia coli isolated in clinical bovine mastitis. Vet. Microbiol. 147:383-388.

Tenaillon, O., D. Skurnik, B. Picard, and E. Denamur. 2010. The population genetics of commensal Escherichia coli. Nat. Rev. Microbiol. 8:207-217.

Thomas, V., A. de Jong, H. Moyaert, S. Simjee, F. El Garch, I. Morrissey, H. Marion, and M. Valle. 2015. Antimicrobial susceptibility monitoring of mastitis pathogens isolated from acute cases of clinical mastitis in dairy cows across Europe: VetPath results. Int. J. Antimicrob. Agents 46:13-20.

Tomazi, T. 2017. Etiological and molecular profile of pathogens causing clinical mastitis, and antimicrobial use in dairy herds. $\mathrm{PhD}$ Thesis. University of Sao Paulo, Pirassununga, Brazil.

Wenz, J. R., F. B. Garry, and G. M. Barrington. 2006. Comparison of disease severity scoring systems for dairy cattle with acute coliform mastitis. J. Am. Vet. Med. Assoc. 229:259-262.

Zhang, D., Z. Zhang, C. Huang, X. Gao, Z. Wang, Y. Liu, C. Tian, W. Hong, S. Niu, and M. Liu. 2018. The phylogenetic group, antimicrobial susceptibility, and virulence genes of Escherichia coli from clinical bovine mastitis. J. Dairy Sci. 101:572-580. 